\title{
Non-traumatic injury profile of amateur cyclists
}

\author{
${ }^{1}$ Section Sports Medicine, University of Pretoria, South Africa \\ ${ }^{2}$ Department of Statistics, University of Pretoria, South Africa \\ ${ }^{3}$ Department of Internal Medicine, University of the Witwatersrand, Johannesburg, South Africa
}

A van der Walt, ${ }^{1} \mathrm{MB}$ ChB; D C Janse van Rensburg, ${ }^{1} \mathrm{MD} ;$ L Fletcher, ${ }^{2} \mathrm{PhD} ; \mathrm{C}$ C Grant, ${ }^{1} \mathrm{PhD} ; \mathrm{A}$ J van der Walt,${ }^{3} \mathrm{FCP}(\mathrm{SA})$

Corresponding author: A van der Walt (altavanderwalt@me.com)

Background. Non-traumatic bicycle injuries are common. However, research available on non-traumatic injuries in amateur cyclists is more than a decade old, and most of the research on this topic has been done in Europe and America on professional cyclists in multi-day cycling events. An understanding of the common injuries may lead to appropriate prevention intervention.

Objective. To determine the incidence of overuse injuries in amateur cyclists preparing for participation in a 1-day cycle challenge. Methods. A questionnaire was emailed to participants of the 2012 Momentum 94.7 Cycle Challenge, which amateur participants $\geq 18$ years old were invited to complete. Data on demographics, training habits and the participants' injury profile in the preceding year were collected. Results. Of the 3300 respondents, 75\% were male and 59\% were between 30 and 50 years old. Non-traumatic injury, pain or neurological symptoms were reported by $88 \%$ of the respondents. The percentages of all respondents who experienced problems in the following anatomical areas were as follows: neck $34 \%$, back $41 \%$, hand/wrist $41 \%$, buttock/perineum $41 \%$, hip $7 \%$, knee $33 \%$ and foot/ankle $24 \%$. Knee pain was responsible for the need to stop training for the largest percentage of respondents. Neurological complaints were common in respondents who experienced neck, back, hand/wrist, buttock/perineum and foot/ankle problems.

Conclusion. Non-traumatic injuries in amateur cyclists are common, with back, hand/wrist and buttock/perineal symptoms the most frequent problems. Knee problems caused the greatest need to stop training and seek medical help.

S Afr J SM 2014;26(4):119-122. DOI:10.7196/SAJSM.555

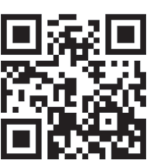

Cycling is a popular sport, in which a large number of amateurs now participate in 1-day events.

The prevalence of non-traumatic bicycle injuries can be as high as $87 \%{ }^{[1,2]}$ The most common injury sites are the knee, neck/shoulder, back, hand/wrist and buttock/ perineum. ${ }^{[1-10]}$ In professional road cyclists, lower back pain and anterior knee pain are the most prevalent overuse injuries, and knee pain is the most likely cause of time lost. ${ }^{[3]}$ Ulnar and median nerve compression appears to be the most common problem in the upper limb. ${ }^{[4-6]}$ One study reported this as an uncommon problem, whereas another study noted that $70 \%$ of cyclists experience either motor or sensory symptoms, or both. ${ }^{[3,4]}$ Neck and shoulder symptoms are common. ${ }^{[1,2]}$ In a study carried out on a 500 -mile, 8 -day bicycle tour, $66.4 \%$ of the cyclists reported some neck or shoulder symptoms, while significant symptoms were reported by $20.4 \% .{ }^{[7]}$ Perineal pain, numbness and erectile dysfunction are complications of prolonged saddle pressure. ${ }^{[11]}$ Twenty-two per cent of male cyclists reported symptoms arising from the innervation area of the pudendal nerve, and impotence was reported by $13 \%{ }^{[8]}$

Much of the current knowledge on overuse cycling injuries comes from cyclists involved in cycle tours $\geq 5$ days, or from professional cyclists attending training camps. ${ }^{[3,4,7,8]}$ Research available on the profile of non-traumatic injuries in amateur cyclists is $>10$ years old. ${ }^{[2,7]}$

South Africa hosts two of the world's largest 1-day cycle challenges, in which most participants are amateurs. Knowledge of the nontraumatic injury profile in these participants is relevant in order to develop appropriate prevention intervention.

The objective of this study was to determine the incidence of overuse injuries in amateur cyclists preparing for a 1-day cycle challenge. This article describes only the injury profile of the cyclists.

\section{Methods}

The Momentum 94.7 Cycle Challenge is a $94.7-\mathrm{km}$ cycle race that takes place in Johannesburg. In 2012, 26331 people entered the event, the vast majority being amateur cyclists.

An e-mail was sent out by the Momentum 94.7 Cycle Challenge race organisers 10 days before the 2012 event, explaining the objective of the study and asking all amateur cyclists $\geq 18$ years to complete a questionnaire via a web link. The questionnaire was voluntary and anonymous; participants had the option to start and then quit the questionnaire at any time. Only questionnaires completed between the time that the e-mail was sent out and the race day were used.

The retrospective questionnaire comprised three parts. The demographic part included general information about the participants that could have affected training and influenced the cyclists' injury profile. The second part focused on training habits. The final part enquired about non-traumatic injuries sustained (including all musculoskeletal and all neurological concerns participants may have experienced due to cycling in the preceding year), and treatment received and time lost. Concerns directly related to falls or accidents were not included. Questions design was based on data from the literature regarding cycling injuries.

The protocol for this study was approved by both the Master of Medicine (MMed) committee and the ethics committee at the Faculty of Health Sciences of the University of Pretoria.

Data analysis comprised frequency tables and cross-tabulations to summarise, and $\chi^{2}$ tests were used to evaluate associations.

\section{Results}

Of the 26331 cyclists who entered the Momentum 94.7 Cycle Challenge, $14 \%$ ( $n=3666)$ followed the link to the survey and $13 \%$ 
( $n=3$ 300) completed the questionnaire. Of the respondents, $75 \%$ ( $n=2476)$ were male and $59 \%(n=1942)$ were between 30 and 50 years old (Fig. 1). These percentages correlated with the general demographics of all participants entering the race.

Non-traumatic injury, pain or neurological symptoms were reported by $88 \%$ ( $n=2904$ ) of the respondents. Problems reported included the neck (34\%, $n=1109)$, back ( $41 \%, n=1340)$, hand/wrist (41\%, $n=1355)$, buttock/perineum (41\%, $n=1358)$, hip (7\%, $n=234)$, knee (33\%, $n=1078)$ and foot/ankle (24\%, $n=782)$.

Significant association was found between sex and injury incidence $\left(\chi^{2}=8.51 ; \mathrm{df}=1\right.$; $p=0.004)$. A negative standardised residual $(-2.4)$ indicated that females are more prone to injuries than males, especially of the neck, knee and foot/ankle (Fig. 2).

No significant association between age and injury susceptibility was found $\left(\chi^{2}=8.25 ; \mathrm{df}=5\right.$; $p=0.14)$. However, the standardised residual (2.3) of respondents $>60$ years showed that substantially more were observed in the no-injury category than expected. Also, no associations were found between age and any specific injuries or combinations of injuries $\left(\chi^{2}=663.48 ; \mathrm{df}=610 ; p=0.07\right)$ (Fig. 3$)$.

Complaint seriousness was assessed by the percentage of participants who needed to stop training, consult a physiotherapist and/or doctor and/or require surgery (Table 1). Knee pain was responsible for the largest percentage (17\%) of respondents who needed to stop training. Few respondents sought help from a doctor and/or physiotherapist. More respondents sought help from a physiotherapist than from a doctor. Four per cent of respondents with knee problems required surgery, while $<1 \%$ of respondents who experienced problems in other anatomical areas needed surgery.

Forty-nine per cent $(n=540)$ of the 1109 respondents who experienced neck pain also experienced neurological symptoms, e.g. numbness or pins and needles in the hands and arms. In $39 \%$ ( $n=211$ ) of these, the neurological symptoms disappeared immediately after cycling. In a further $37 \%(n=202)$ they disappeared within $1 \mathrm{~h}$ after cycling, while $5 \%(n=25)$ experienced the symptoms for $>1$ week after cycling.

Twenty per cent $(n=261)$ of the 1340 respondents who experienced back pain experienced neurological symptoms, e.g. pins and needles or numbness in their legs. In $34 \%(n=88)$ of these, symptoms disappeared immediately after cycling. In a further $35 \%(n=91)$, the symptoms

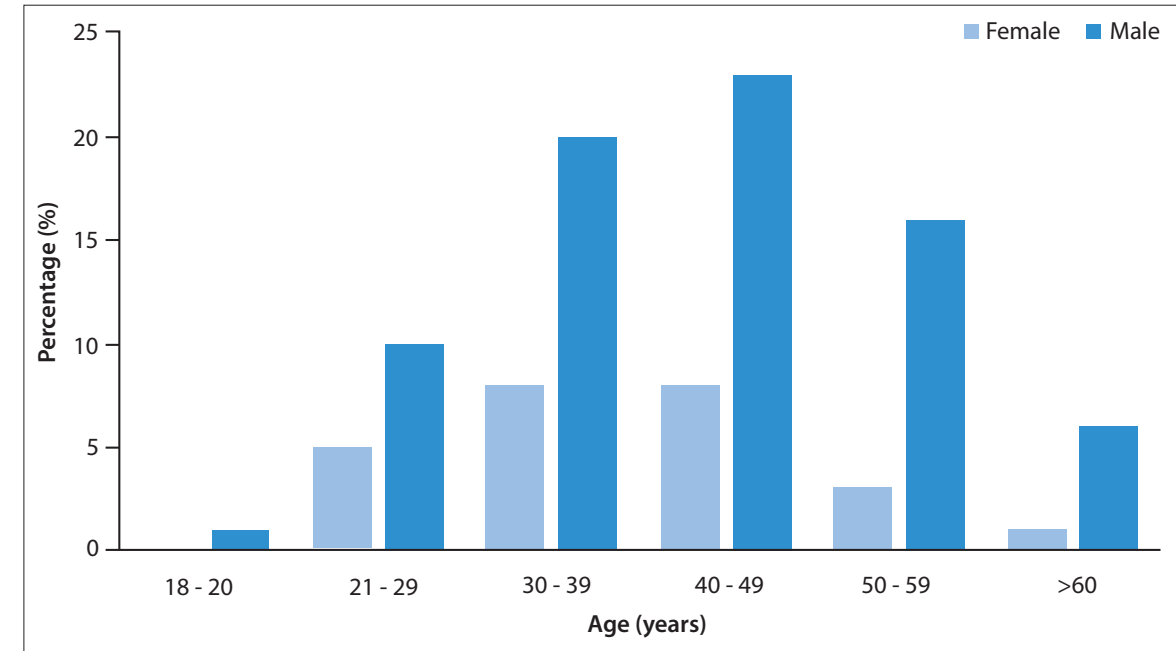

Fig. 1. Age and sex distribution of respondents.

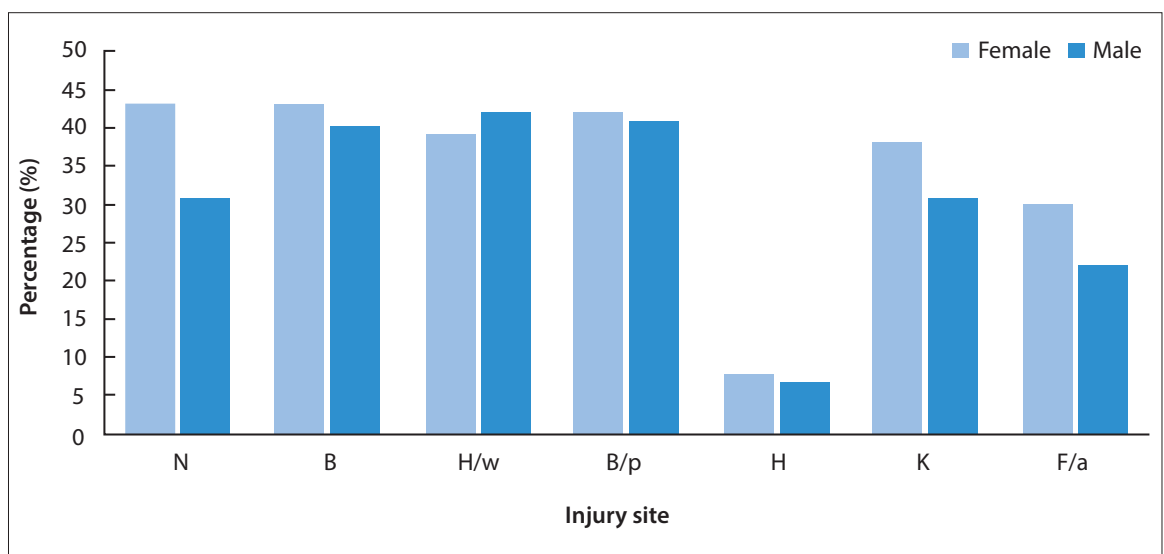

Fig. 2. Percentage of all male $v$. female respondents that reported pain or other symptoms in the different anatomical areas. $(N=$ neck; $B=$ back; $H / w=$ hand $/$ wrist $B / p=$ buttock/perineum; $H=$ hip; $K=$ knee; $F / a=$ foot/ankle. $)$

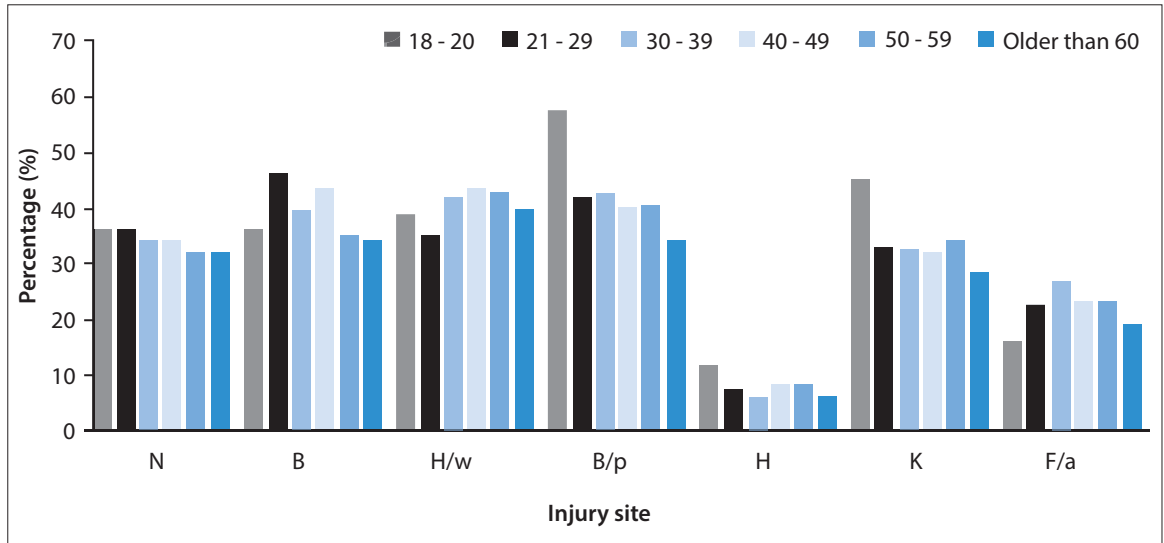

Fig. 3. Percentage of respondents in the different age groups that experienced pain or other symptoms in the different anatomical areas. $(N=$ neck; $B=$ back; $H / w=h a n d /$ wrist; $B / p=$ buttock/perineum; $H=$ hip; $K=$ knee; $F / a=$ foot/ankle. $)$

lasted $<1 \mathrm{~h}$ after cycling, while in $10 \%(n=26)$, these symptoms lasted for $>1$ week.

Wrist symptoms were reported by $41 \%$ ( $n=1355)$ of respondents. Of these, 23\%
( $n=307)$ had pain, 67\% ( $n=909)$ had pins and needles, 44\% ( $n=595)$ had loss of sensation and $13 \%(n=176)$ had weakness. Some respondents had more than one symptom. In $85 \%$ 
( $n=1$ 155), these symptoms disappeared within $1 \mathrm{~h}$ after cycling. Symptoms were experienced in both hands by $64 \%(n=867)$ of respondents with wrist concerns, and in $20 \%(n=271)$ only in the left hand and $16 \%(n=217)$ only in the right hand.

Of the 1358 respondents who complained of symptoms in the buttock/perineal area, 53\% $(n=724)$ had pain, 49\% ( $n=649)$ had loss of sensation, 25\% $(n=339)$ had pins and needles and $3 \%(n=40)$ experienced erectile dysfunction. Some experienced more than one symptom. In $70 \%(n=947)$, these symptoms disappeared within $1 \mathrm{~h}$ of cycling. However, erectile dysfunction is a notable exception. Only $0.02 \%(n=40)$ of males experienced erectile dysfunction, but in $20 \%(n=8)$ of these, the problem endured for $>1$ week.

Although only 7\% ( $n=234)$ of respondents experienced hip pain, the symptoms lasted longer than for other injuries. In $18 \%(n=43)$, the symptoms lasted $>1$ week, while another $19 \%(n=44)$ had pain for $>1$ day but $<1$ week, and 20\% $(n=46)$ had pain for $>1$ h but $<1$ day. Thus, $57 \%$ ( $n=133)$ had pain enduring for $>1 \mathrm{~h}$.

Knee problems were reported by $33 \%$ $(n=1078)$. Of these, $56 \%(n=600)$ experienced pain in the anterior area of the knee, $37 \%$ $(n=398)$ on the lateral side, $18 \%(n=179)$ medially and $6 \%(n=67)$ posteriorly. On the questionnaire, respondents could choose more than one anatomical area to indicate where problems were experienced. Knee pain lasted $>1$ week in $14 \%(n=85)$ of respondents with anterior knee pain, $19 \%(n=73)$ with lateral knee pain, 19\% ( $n=37)$ with medial knee pain and $15 \%(n=10)$ with posterior knee pain.

Foot and ankle problems were noted by $24 \%(n=782)$. Of these, $60 \%(n=469)$ reported numbness of the foot, $47 \%(n=366)$ pins and needles of the foot, $20 \%(n=155)$ pain in the foot, $9 \%(n=68)$ pain in the ankle, and only $3 \%$ $(n=23)$ weakness of the foot. Some respondents experienced more than one symptom. In $45 \%(n=489)$, the symptoms disappeared immediately after cycling, and in a further $35 \%$ $(n=381)$, it disappeared within $1 \mathrm{~h}$ after cycling.

\section{Discussion}

Eighty-eight per cent ( $n=2904)$ of respondents complained of a non-traumatic injury, pain or neurological symptoms. This finding agrees with data that found that non-traumatic injuries in cyclists can be as high as $87 \%{ }^{[1,2]}$ However, not all pain or other symptoms in this study were necessarily related to a nontraumatic cycling injury. An individual history and clinical examination were not carried out. Respondents were asked only whether they experienced pain or other symptoms not due to trauma.

Back pain, hand/wrist problems (pain, numbness or weakness) and buttock/perineal pain or numbness were the most common problems cyclists experienced (41\% each). Many respondents also experienced neck pain (34\%) and knee pain (33\%). Foot/ankle problems were less common (24\%). Only $7 \%$ experienced hip problems. Wilber et al. ${ }^{[2]}$ also made use of a questionnaire to assess overuse injuries in amateur cyclists, and found neck injuries/ complaints to be the most common (49\%), followed by knees (42\%), groin/buttock (36\%), hands (31\%) and back (30\%). Bicycles, cycling gear and emphasis on bike setup have developed since the publication of Wilber et al's study in 1995, which may account for some of the differences in the results between Wilber et al.'s study and the current study. A review article on non-traumatic bicycle injuries found the prevalence of injuries to the knee to be $22-64 \%$, neck $3-49 \%$, hand/wrist $10-70 \%$, buttocks/ perineum $9-61 \%$, back $15-50 \%$, upper leg/hip 2 - 25\%, and lower leg/foot $7-22 \% .{ }^{[3]}$ Although most of these studies were done on multi-day cycle events, our data fit well with these findings.
In evaluating the seriousness of the problem in terms of the need to stop training, knee injuries appeared to be the most serious, with $17 \%$ of respondents with knee problems needing to stop training. This finding correlates with research done on professional road cyclists. ${ }^{[3]}$ After knee injuries, in order of decreasing frequency, the need to stop training was highest in hip-related problems (13\%), back-related problems (9\%), neckrelated problems (5\%), foot/ankle-related problems (4\%), hand/wrist-related problems (2\%) and finally buttock/perineum-related problems (1\%). Even though buttock/perineal problems had a high incidence $(41 \%)$ very few needed to stop training. Weiss ${ }^{[7]}$ reported knee and neck/shoulder problems the most common areas with symptoms severe enough to stop cycling.

Hip and knee injuries accounted for the most doctor visits, whereas neck and back injuries had the highest percentage of physiotherapy consultations. Knee injuries were responsible for the largest number of cyclists receiving medical treatment in Weiss's study. ${ }^{[7]}$

Four per cent of respondents with knee pain required surgery. In all the other anatomical areas, $<1 \%$ of the respondents who experienced pain needed surgery. No study could be found for comparison with the current study.

An association was found between sex and the probability of sustaining an injury in general $(p=0.004)$. However, this association could have been influenced by the large sample size. In the current study, females were more prone to injuries than males. The specific injury profiles showed that females are more inclined to suffer neck, knee and foot/ankle injuries. Although Wilber et al. ${ }^{[2]}$ found no significant difference between male and female injuries in the various anatomical sites, they reported that female cyclists complained 1.5 times more than male cyclists about neck problems.

Table 1. Respondents' problems and treatment sought

\begin{tabular}{|c|c|c|c|c|c|}
\hline Area & $\begin{array}{l}\text { Needed to stop } \\
\text { training, } n(\%)\end{array}$ & $\begin{array}{l}\text { Sought help from a doctor } \\
\text { and a physiotherapist, } n(\%)\end{array}$ & $\begin{array}{l}\text { Sought help from } \\
\text { a doctor, } n(\%)\end{array}$ & $\begin{array}{l}\text { Sought help from a } \\
\text { physiotherapist, } n(\%)\end{array}$ & $\begin{array}{l}\text { Needed surgery, } \\
n(\%)\end{array}$ \\
\hline Neck & $53(5)$ & $83(8)$ & $121(11)$ & $251(23)$ & $3(0.3)$ \\
\hline Back & $121(9)$ & $94(7)$ & $160(12)$ & $297(22)$ & $6(0.4)$ \\
\hline Hand/wrist & $24(2)$ & $18(1)$ & $50(4)$ & $47(4)$ & $5(0.4)$ \\
\hline Buttock/perineum & $43(1)$ & $6(0.2)$ & $37(1)$ & $29(1)$ & $6(0.2)$ \\
\hline Hip & $31(13)$ & $22(9)$ & $39(17)$ & $50(21)$ & $1(0.4)$ \\
\hline Knee & $178(17)$ & $108(10)$ & $185(17)$ & $222(21)$ & $40(4)$ \\
\hline Foot/ankle & $30(4)$ & $21(3)$ & $62(8)$ & $37(5)$ & $2(0.3)$ \\
\hline
\end{tabular}


No statistical association between age and injuries was found. It is, however, noticeable that the 18 - 20-year age group had a proportionally higher incidence of buttock/perineal, hip and knee problems than other age groups (Fig. 3). Less than $1 \%(n=31)$ of the respondents belonged to this age group; for this reason, this finding is of doubtful significance. No other studies could be found that compared age and cycling injuries. It is interesting that fewer than expected respondents in the $\geq 60$ years age group sustained any injuries. This could possibly be attributed to years of cycling experience, since $55 \%$ of this age group had been participating in events for $>10$ years and a further $24 \%$ had been participating in events for 5 - 10 years.

Neurological symptoms were common. Thirty-seven per cent ( $n=1241)$ of respondents reported neurological symptoms in the wrists and hands. This finding correlates well with Wilber et al.'s ${ }^{[2]}$ study, which reported hand symptoms in $31 \%$ of recreational cyclists. Patterson et al. ${ }^{[4]}$ found neurological symptoms in $70 \%$ of cyclists who participated in a $600-\mathrm{km}$ ride. However, participants taking part in a $600-\mathrm{km}$ ride would cycle more kilometres when training than the average amateur cyclist training for a 100-km cycle challenge, which may account for the difference in percentages. Nerve conduction studies on cyclists before and after a 6-day bicycle tour found that long-distance cycling may promote physiological changes in the deep branch of the ulnar nerve. Long-distance cycling may also exacerbate symptoms of carpal tunnel syndrome. ${ }^{[12]}$ The high prevalence of neurological hand symptoms found in our study indicates that ulnar and median nerve palsy are not only a problem among professional cyclists participating in multi-day cycle events, but are also found in amateur cyclists who participate in 1-day events. Slane et al. ${ }^{[13]}$ found that padded gloves and changing hand position can decrease the pressure applied to the ulnar and median nerves.

Eighty-four per cent $(n=655)$ of respondents with foot/ankle problems had neurological symptoms such as pins and needles, weakness and loss of sensation. Wanich et al. ${ }^{[10]}$ reported that increased stiffness of cycling shoes may provide more efficient energy transfer but that it also increases forefoot pressure. Of the 1358 respondents with buttock/ perineal complaints, $62 \%(n=842)$ had neurological symptoms. These symptoms abated mostly within $1 \mathrm{~h}$ after cycling had stopped, except in the small percentage $(0.02 \%)$ of males who experienced erectile dysfunction. Leibovitch and Mor ${ }^{[11]}$ reviewed bicycling-related urogenital disorders and reported erectile dysfunction in $13-24 \%$ of cyclists, and urological complaints and nerve entrapment syndromes in $50-91 \%$. Our findings differ substantially; perhaps, as a result of underreporting of this sensitive subject in our study. The difference between our findings and those by Leibovitch and Mor might also be due to the fact that amateur cyclists training for a 1-day event spend a lot less time on a bicycle than professional cyclists and cyclists involved in multi-day events. A selection bias in the article by Leibovitch and Mor should also be considered, as the focus was on genito-urinary complaints in cyclists. Forty-nine per cent $(n=540)$ of the respondents with neck complaints and $20 \%(n=261)$ of the respondents who reported back pain also reported neurological symptoms in the arms and legs. Manninen and Kallinen ${ }^{[14]}$ reported neurological symptoms in the legs associated with lower back pain in $32 \%$ of the 92 triathletes who reported lower back pain. No other studies investigating the relationship between neurological symptoms related to neck and back pain in cyclists could be found.

This study was based on a retrospective questionnaire, which has the advantage of providing access to a large sample size. However, the study has certain limitations, such as recall bias and volunteer bias. In order to make specific diagnoses, clinical examinations and further investigations would be needed.

\section{Conclusion}

Non-traumatic injuries, pain not due to trauma and neurological symptoms are common in amateur cyclists training for a 1-day cycle challenge. Back pain, hand/wrist symptoms and buttock/perineal pain or numbness were the most common problems cyclists experienced, followed by neck pain, knee pain, foot/ankle problems and, lastly, hip problems. Knee pain appears to be the problem of most concern in amateur cyclists. Compared with other anatomical areas, a disproportionate number of cyclists with knee problems needed to stop training, visit a doctor and require surgical intervention. Neurological symptoms were experienced by a large percentage of respondents who experienced neck and back pain, hand/wrist symptoms and buttock/ perineal symptoms.

Very little current research is available on non-traumatic injuries in amateur cyclists. The objective of this study was not to identify specific cycling injuries but rather to determine the extent and seriousness of non-traumatic injuries, pain or neurological dysfunction in anatomical areas that are believed to be potential high-risk injury areas in cyclists. The information from this study can therefore be used as a foundation to guide future research to investigate the causes of non-traumatic injuries in cyclists, and may direct interventions that help with the prevention of non-traumatic injuries in cyclists.

\section{References}

1. Dettori NJ, Norvell DC. Non-traumatic bicycle injuries: A review of the literature. Sports Med 2006;36(1):7-18. [http://dx.doi.org/10.2165/00007256-200636010-00002]

2. Wilber CA, Holland GJ, Madison RE, Loy SF. An epidemiological analysis of overuse injuries among recreational cyclists. Int J Sports Med 1995;16(3):201-206. [http:// dx.doi.org/10.1055/s-2007-972992]

3. Clarsen B, Krosshaug T, Bahr R. Overuse injuries in professional road cyclists. Am J Sports Med 2010;38(12):2494-2501. [http://dx.doi.org/10.1177/0363546510376816]

4. Patterson JM, Jaggars MM, Boyer MI. Ulnar and median nerve palsy in long-distance cyclists. Am J Sports Med 2003;31(4):585-589. [http://dx.doi.org/10.1177/036354650 30310041801]

5. Mellion MB. Common cycling injuries: Management and prevention. Sports Med 1991;11(1):52-70. [http://dx.doi.org/10.2165/00007256-199111010-00004]

6. Dannenberg AL, Needle S, Mullady D, Kolodner KB. Predictors of injury among 1638 riders in a recreational long-distance bicycle tour: Cycle across Maryland. Am J Sports Med 1996;24(6):747-753. [http://dx.doi.org/10.1177/036354659602400608]

7. Weiss BD. Non-traumatic injuries in amateur long distance bicyclists. Am J Sports Med 1985;13(3):187-192.

8. Anderson KV, Bovim G. Impotence and nerve entrapment in long distance amateur cyclists. Acta Neurol Scand 1997;95(4):233-240. [http://dx.doi.org/10.1111/j.1600-0404.1997. tb00104.x]

9. Cohen GC. Cycling injuries. Can Fam Physician 1993;39:628-632.

10. Wanich T, Hodgkins C, Columbier JA, Muraski E, Kennedy JG. Cycling injuries of the lower extremity. J Am Acad Orthop Surg 2007;15(12):748-756.

11. Leibovitch I, Mor Y. Review. The vicious cycling: Bicycling related urogenital disorders. Eur Urol 2005;47(3):277-286. [http://dx.doi.org/10.1016/j.eururo.2004.10.024]

12. Akuthota V, Plastaras C, Lindberg K, Tobey J, Press J, Garvan C. The effect of long-distance bicycling on ulnar and median nerves: An electrophysiologic evaluation of cyclist palsy. Am J Sports Med 2005;33(8):1224-1230. [http://dx.doi. org/10.1177/0363546505275131]

13. Slane J, Timmerman M, Ploeg HL, Thelen DG. The influence of glove and hand position on pressure over the ulnar nerve during cycling. Clin Biomech (Bristol, Avon) 2011;26(6):642-648. [http://dx.doi.org/10.1016/j.clinbiomech.2011.03.003]

14. Manninen JSO, Kallinen M. Low back pain and other overuse injuries in a group of Japanese triathletes. Br J Sports Med 1996;30(2):134-139. [http://dx.doi.org/10.1136/bjsm.30.2.134] 